\title{
A Problem Not to Be Born Into
}

\author{
Guarina Isabel Molina* \\ Universidad Ibero americana (UNIBE), Dominican Republic
}

*Corresponding author: Guarina Isabel Molina, Universidad Ibero americana (UNIBE), Dominican Republic.

To Cite This Article: Guarina Isabel Molina. A Problem Not to Be Born Into. Am J Biomed Sci \& Res. 2019 - 4(5). AJBSR.MS.ID.000824. DOI: 10.34297/AJBSR.2019.04.000824

Received: August 14, 2019 | Published: August 19, 2019

\section{Editorial}

In adults, obesity is defined as having a body mass index (BMI) over $30 \mathrm{~kg} / \mathrm{m}^{2}$, and as of 2016,13\% of adults worldwide suffered from obesity. While being a preventable condition, the prevalence worldwide continues to rise year after year. On this instance, I would like to take a word to focus my attention of a special population group in which this epidemic promise to do twice the harm: pregnant women. When thinking of a pathology at hand, one is usually concerned about the health of the mother. However, more recent studies have pointed out evidence that maternal obesity is not only a threat to the gestating woman, but also to the offspring, both in early childhood and in adult life. Is it to wonder if, by not educating ourselves and our patients on time, we are allowing our future generations to be born into a culture of disease. Statistics report that over 14 million women worldwide in 2014 suffered from obesity, and that over $1 / 3$ of pregnancies in the United States occurred on obese women.

In 2016, The Lancet: Diabetes \& Endocrinology published a 4-part series concerning maternal obesity [1], highlighting through several angles the impending danger that obesity, before and during pregnancy, as well as the post-partum period [2], represent on both the mother and offspring. This series was published almost 3 years ago, calling attention to this public health problem affecting women and children worldwide. Yet it seems that even with strong supporting evidence, the effort made as individuals, professionals and patients alike, hasn't had enough grip to slow the rise of this inevitably growing epidemic. Stigma placed on weight has led many patients to believe that the emphasis made by healthcare professionals on carrying pregnancy with a healthy weight is just an opinion, or personal preference. Data shows that both parties, mother and child alike, were under the effects of maternal obesity, as well as the scientific biological mechanisms proposed for the effects on the child. Obesity can have long term effects, and it constitutes a public health problem that is not unique to a nation, it's affecting our mothers and our children throughout the world.
The third article of the Maternal Obesity series, entitled "Influence of maternal obesity on the long-term health of the offspring" provides solid data on the complications that can arise during pregnancy due to maternal obesity and its impact on the health of the product [3]. The article explains the consequences for the offspring on several areas, highlighting an increase in childhood obesity, relation to poor cardiovascular health and diabetes in adulthood, rising prevalence of asthma and wheezing, as well as alterations in gut microbiome and further decline on immune health. It is yet to be investigated if weight during pregnancy has any effects on the neurobehavioral health of the child or if this exposure is of detriment during any specific period of pregnancy. While the causality of such epidemic is not clear, it cannot be ignored that having a stable and healthy pre-pregnancy weight helps the gestating woman to have better control during pregnancy, that is, in of itself, a period of great change. The effects of obesity on adults are widely known, and as grown individuals we have the power, and moreover, the responsibility to take our health into our hands [4].

Pregnancy is not a disease, it's a physiological process that could be complicated by multiple factors, some preventable, and others inevitable. The purpose of working as a team in achieving good health for the mother and a baby is not about placing shame, but about facing the fact that it is our duty, both of patients and health professionals, to make this process as easy as can be. To have, in the end, not just healthy mothers, but also healthy babies who can grow into healthy children and healthy adults and spare our children from the burden of risk and disease since such a young age.

\section{References}

1. Godfrey KM, Reynolds RM, Prescott SL, Nyirenda M, Jaddoe VW, et al. (2017) Influence of Maternal Obesity on the Long-Term Health of Offspring. Lancet Diabetes and Endocrinology 5(1): 53-64.

2. Wan Ma RC, Schmidt MI, Tam WH, Mclntyre HD, Catalano PM (2016) Clinical Management of Pregnancy in the Obese Mother: before Conception, during Pregnancy, and Post Partum. Lancet Diabetes and Endocrinology 4: 12 . 
3. Hanson M, gluckman P, Bustreo F (2016) Obesity and the Health of Future Generations. Lancet Diabetes \& Endocrinology 4: 12

4. World Health Organization (2018) Obesity and Overweight. 